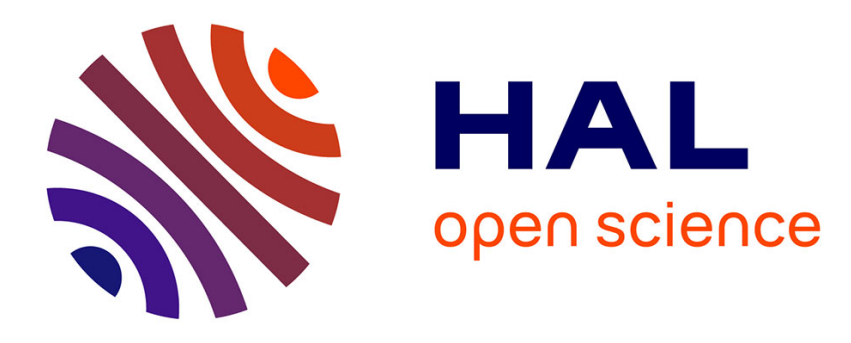

\title{
Crise et réorganisation des Alpes Slovènes depuis l'indépendance
}

Angela Barthes

\section{To cite this version:}

Angela Barthes. Crise et réorganisation des Alpes Slovènes depuis l'indépendance. Méditerranée: revue géographique des pays méditerranéens, 2004, n³ 3-4, pp 31-36. halshs-00007851

\section{HAL Id: halshs-00007851 https://shs.hal.science/halshs-00007851}

Submitted on 17 Jan 2006

HAL is a multi-disciplinary open access archive for the deposit and dissemination of scientific research documents, whether they are published or not. The documents may come from teaching and research institutions in France or abroad, or from public or private research centers.
L'archive ouverte pluridisciplinaire $\mathbf{H A L}$, est destinée au dépôt et à la diffusion de documents scientifiques de niveau recherche, publiés ou non, émanant des établissements d'enseignement et de recherche français ou étrangers, des laboratoires publics ou privés. 


\title{
CRISE ET REORGANISATION DES ALPES SLOVENES DEPUIS L'INDEPENDANCE
}

\author{
BARTHES Angela, UMR 6570 TELEMME, MMSH, 13094 Aix en Provence
}

Résumé $^{1}$ : Les mutations sociales rapides des Alpes slovènes liées, entre autres, à l'entrée en concurrence des unités de production traditionnelles, se traduisent par une réorientation fonctionnelle des territoires et une redistribution spatiale de la population. Le rôle relatif de l'agriculture et de l'industrie décroît au profit des activités de service. L'effondrement productif et la chute brutale du nombre d'exploitations accroissent le taux de chômage et la fréquence des migrations pendulaires vers les villes de plaines.

\begin{abstract}
:
Mots clefs : Montagne, Agriculture, Slovénie, Recensement, Population, Tourisme

Key words: Mountains, Slovenia, Agriculture, Census, Population, Tourism
\end{abstract}

\section{Introduction :}

La République Slovène, l'un des plus jeunes Etats d'Europe, s'étend sur $20256 \mathrm{~km}^{2}$ et compte deux millions d'habitants. Accrochée à l'est de l'Arc alpin, l'altitude moyenne est élevée et plus du tiers du territoire se trouve au dessus de 600 mètres. La zone d'étude -la région alpine et les préalpes- frontalière de l'Italie et de l'Autriche, est située au nord-ouest du pays et s'étend sur 53 municipalités (Carte 1).

Dans la région alpine, le gradient altitudinal est important, variant de 178 mètres dans les vallées fluvio-glaciaires à 2864 mètres au sommet du mont Triglav. Les conditions topographiques et édaphiques constituent un facteur limitant pour l'agriculture : hormis les surfaces de calcaires nus, une grande partie du territoire est couvert de forêts. Comme dans la plupart des hautes montagnes alpines, l'élevage est une activité très développée, et la surface des alpages est importante. Le principal revenu agricole provient des ovins et des bovins qui se répartissent uniformément sur le territoire. La région centrale, comprend six municipalités ${ }^{2}$ intégrées au parc national du Triglav dans laquelle d'importants efforts en direction du tourisme sont accomplis.

La région préalpine est le domaine de la moyenne montagne. Elle est principalement couverte de forêts. Très compartimentée en vallées étroites, le semis urbain est irrégulier et les villages peu accessibles. L'agriculture et l'industrie se concentrent dans les parties les plus basses. Le tourisme est peu développé, et se manifeste presque exclusivement en été, à proximité des lacs et rivières destinés à la pêche et à la baignade.

Depuis l'indépendance du jeune Etat, intervenue en 1991, les espaces alpins font face à de profonds bouleversements. Les effondrements productifs agricoles et la rupture de fonctionnement des centres industriels entraînent une nouvelle répartition des activités économiques. Aujourd'hui, $25 \%$ de la population active travaille dans l'agriculture et $12 \%$ dans le secteur industriel, contre respectivement $43 \%$ et $38 \%$ en 1990. La vie sociale dans les espaces ruraux de montagne s'en trouve ainsi profondément modifiée.

\footnotetext{
${ }^{1}$ Je remercie Régis Darques pour son aide précieuse

2 Kranska Gora, Jesenice, Radovljica, Bled, Bohinj et Bovec
} 


\title{
I. Crise agricole et effondrement productif en montagne
}

\author{
1. Une réorganisation globale de l'agriculture défavorable à la \\ région alpine.
}

A l'échelle nationale, la réorganisation de l'agriculture se manifeste par une plus grande spécialisation des régions les plus productives. Ainsi, l'Est du Pays, moins compartimenté, voit sa part de surface cultivée augmenter et les cultures sont soumises à l'intensification générale avec l'apparition de nombreux tracteurs modernes (Carte 2). En contrepartie, les exploitations montagnardes déclinent, et leur emprise spatiale régresse. Ces faits sont associés à un effondrement des productions animales et végétales (Petek F., 2002; Vrišer I., 2002). Seule fait exception la vallée irriguée de la Soca, parallèle à la frontalière italienne, où la moitié des terres est encore maintenue en culture notamment au nord, à proximité de Bovec.

Ce processus lié à la transition vers l'économie de marché a débuté au milieu des années 70 et n'a cessé de s'accélérer depuis l'indépendance. La décollectivisation ${ }^{3}$ qui s'est opérée de 1991 à 1996 n'a eu que peu d'impact. Seulement 40\% des terres collectives ont été redistribuées aux anciens propriétaires, ce qui représente à peine 3\% des terres cultivées. L'abrogation en 1991 de la loi sur la propriété foncière de $1953^{4}$ qui limitait les surfaces maximales autorisées dans les fermes individuelles, a en revanche considérablement modifié le visage des exploitations agricoles. Concrètement, cette mesure permet aux exploitations les plus prospères de s'étendre, par rachat des structures les moins compétitives. Le processus de concentration déjà à l'œuvre de longue date dans les pays d'Europe de l'Ouest prend alors son essor.

Le fait marquant reste la contraction importante du nombre d'exploitants (Carte 3). Le phénomène se traduit en plaine par l'intensification des productions (Tableau 1) et le regroupement de la propriété. En montagne au contraire, elle se traduit par un certain abandon de l'espace pastoral, la progression des friches et de la forêt (Meze D., 1987).

Tableau 1 : L'importante augmentation de la surface agricole utilisée moyenne par exploitation entre 1991 et 2000 en Slovénie.

\begin{tabular}{|l||c||c||c|}
\hline & $\begin{array}{c}\mathbf{1 9 9 1} \\
\text { (en hectares) }\end{array}$ & $\begin{array}{c}\mathbf{2 0 0 0} \\
\text { (en hectares) }\end{array}$ & $\begin{array}{c}\text { Evolution } \\
\mathbf{1 9 9 1 - 2 0 0 0}\end{array}$ \\
\hline \hline Moyenne & 2,72 & 5,51 & $109,6 \%$ \\
\hline \hline Ecart Type & 0,83 & 1,6 & $58,7 \%$ \\
\hline
\end{tabular}

D'un point de vue social, la disparition de la moitié des exploitations de l'espace alpin en dix ans, entre 1991 et 2001, met au chômage 60\% des agriculteurs (Tableau 2). La plupart d'entre eux ont aujourd'hui de grandes difficultés de reconversion et les plus âgés poursuivent leurs activités à des fins d'autoconsommation.

\footnotetext{
${ }^{3}$ Afin de faciliter le transfert des coopératives agricoles vers le secteur privé, est constituée le 30 mai 1993 une caisse pour les terres agricoles et forestières. A l'indépendance, à l'inverse des autres pays de l'Europe de l'Est (excepté la Pologne), 92\% des terres cultivées appartenaient à de petits propriétaires, tandis que le secteur collectif représentait à peine $8 \%$. Ce ratio était encore plus fort en montagne.

${ }^{4}$ La loi sur la propriété foncière est votée par le gouvernement de la fédération yougoslave en mai 1953 et stipule que l'ensemble des terres labourées appartenant à des fermes individuelles ne doit pas dépasser 10 hectares, ou 15 hectares dans certains cas.
} 
Tableau 2 : La baisse du nombre moyen d'exploitants agricoles par municipalité dans les régions de montagne entre 1991 et 2000

\begin{tabular}{|l||c|c|c|}
\hline & $\mathbf{1 9 9 1}$ & $\mathbf{2 0 0 0}$ & $\begin{array}{c}\text { Evolution } \\
\mathbf{1 9 9 1 - 2 0 0 0}\end{array}$ \\
\hline \hline Zone alpine & 495 & 299 & $-60,4 \%$ \\
\hline Préalpes & 265 & 150 & $-56,6 \%$ \\
\hline
\end{tabular}

\section{Des exploitations résiduelles extensives et polycoles}

Pénalisées par des surfaces exiguës, les exploitations restantes sont très fragiles. La pratique de la polyculture sur des surfaces aussi réduites, révèle un système peu productif (Vrišer I, 2002). En effet, plus des deux tiers des fermes se contentent de surfaces cultivées inférieures à 10 hectares et certaines se réduisent à trois vaches et quelques champs d'herbes fourragères associées (Tableaux 3 et 4).

Tableau 3 : Occupation du sol en région alpine

\begin{tabular}{|c|c|c|}
\hline Type d'occupation du sol & Pourcentage de la surface totale & Hectares \\
\hline Surface cultivée & 18,2 & 51026 \\
\hline Forêt & 59,8 & 167937 \\
\hline Pâturages & 12,3 & 34484 \\
\hline Terrains non fertiles & 9,7 & 27195 \\
\hline Total & $\mathbf{1 0 0}$ & $\mathbf{2 8 0 6 4 2}$ \\
\hline
\end{tabular}

Source : Office statistique slovène 2002

Tableau 4 : Les productions végétales et animales en région alpine

\begin{tabular}{|c|c|c|c|}
\hline $\begin{array}{c}\text { Type de production } \\
\text { Végétale }\end{array}$ & $\begin{array}{l}\text { Pourcentage de la } \\
\text { surface cultivée }\end{array}$ & $\begin{array}{c}\text { Type de production } \\
\text { Animale }\end{array}$ & $\begin{array}{l}\text { Pourcentage du } \\
\text { nombre de têtes }\end{array}$ \\
\hline Fourrage & 88 & Bovins & 82,1 \\
\hline Céréales & 7,3 & Porcins & 12,7 \\
\hline $\begin{array}{c}\text { Citrouilles, Betteraves, } \\
\text { Pommes de terres }\end{array}$ & 4,3 & Equins & 2,3 \\
\hline Vergers & 0,2 & Ovins & 1,6 \\
\hline Vignobles & 0,1 & Volailles & 0,9 \\
\hline Autres & 0,1 & Autres & 0,1 \\
\hline & \multicolumn{2}{|c|}{ Surface cultivée totale } & e de têtes total \\
\hline & \multicolumn{2}{|c|}{$51026 \mathrm{ha}$} & 97106 \\
\hline $\begin{array}{c}\text { Nombre } \\
\text { d'exploitations }\end{array}$ & \multicolumn{2}{|c|}{63782} & 12138 \\
\hline $\begin{array}{c}\text { Moyenne par } \\
\text { exploitation }\end{array}$ & \multicolumn{2}{|c|}{0,8 hectares } & 8 têtes \\
\hline
\end{tabular}

Source : Office statistique slovène 2002

Aujourd'hui, la production agricole ne suffit plus à subvenir aux besoins de la population locale. La pluriactivité est devenue prépondérante : les conditions altitudinales et édaphiques défavorables, la taille des exploitations insuffisante, l'entrée dans l'économie de marché, explique le recours à des revenus complémentaires. Moins d'une exploitation sur deux assure 
ses revenus de l'agriculture seule, et dans la zone touristique du mont Triglav, il n'y en reste plus que $20 \%$.

Si les conditions d'exercice sont aujourd'hui difficiles, l'avenir s'avère encore plus sombre: dans toutes les régions de montagne, la relève des exploitations n'est assurée que dans $40 \%$ des cas. De plus, l'âge moyen des exploitants n'a cessé de progresser et plus de la moitié de la population agricole dépasse 55 ans. On compte un quart de retraités. Plusieurs municipalités du nord-ouest sont dépourvues d'exploitants de moins de 45 ans (Kobarid, Kranjska Gora). En effet, la rentabilité trop faible face à la nouvelle concurrence, l'accessibilité médiocre, la crise des élevages traditionnels et des équipements collectifs trop lacunaires accélèrent le départ des jeunes agriculteurs. Les nouveaux exploitants s'installent de préférence dans les régions les plus basses. La situation est un peu différente dans le parc du Triglav, où l'âge moyen s'abaisse légèrement, en raison de la pénibilité du travail, qui pousse à transmettre l'exploitation à ses enfants dans le cadre des incitations à l'installation des jeunes agriculteurs pluriactifs (tourisme et agriculture). Par ailleurs, le niveau de formation des agriculteurs est faible avec seulement $5 \%$ des exploitants diplômés du secteur. Ce manque de formation est un frein au développement de l'activité agricole et à l'optimisation des productions.

\section{De la crise agricole à la réorganisation de l'espace rural montagnard}

\section{Des mutations fonctionnelles fondamentales mais défavorables à l'emploi}

Jusque dans les années 1980, l'agriculture employait une main d'œuvre importante, du fait de la multitude des exploitations de petite taille. Cet état de fait, lié à la loi sur la propriété foncière, protège la montagne du dépeuplement en ralentissant l'émigration vers les plaines. Les villages montagnards sont donc maintenus dans une situation économique et démographique relativement favorable, en regard d'autres régions similaires d'Europe occidentale. La disparition d'une exploitation agricole sur deux et l'effondrement industriel parallèle ont modifié l'équilibre du marché local de l'emploi tout en initiant une forte réorientation fonctionnelle et spatiale de la montagne (Tableaux 5).

Tableau 3 : Evolution de l'emploi en montagne : Vers une reconversion fonctionnelle, 1989-2003 


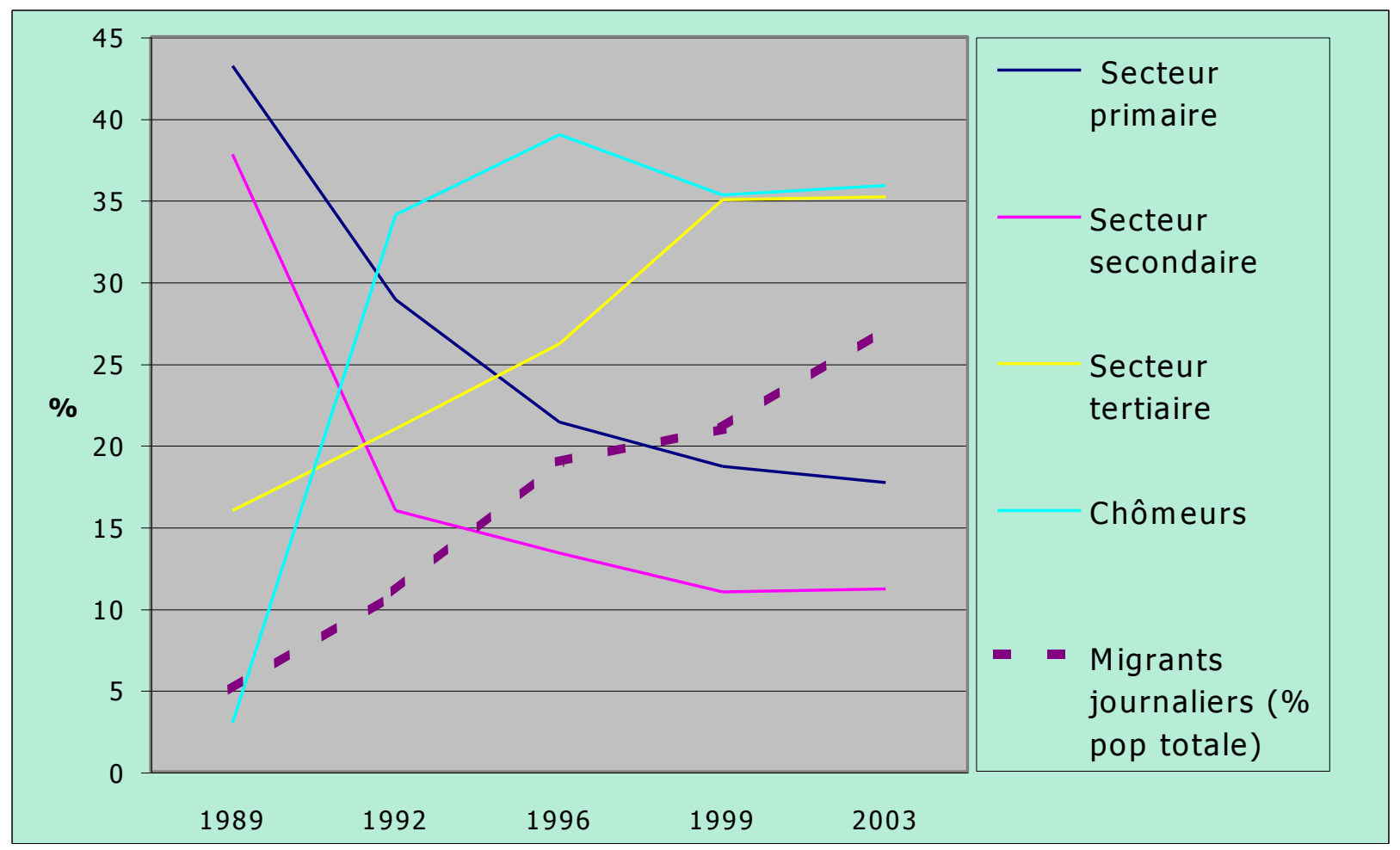

La perte sèche du nombre d'emplois est estimé en montagne entre $-10 \%$ et $-30 \%$ en dix ans selon les sources, avec au moins trois conséquences: l'accroissement du chômage, la précarisation d'une partie de la population dans un contexte de hausse généralisée du niveau de vie, une croissance soutenue des migrations journalières avec près d'un montagnard sur quatre se rend quotidiennement à Ljubljana ou dans les villes intermédiaires des plaines alpines pour y travailler.

Le tourisme et activités de loisir viennent désormais soutenir les régions de haute montagne ( $10 \%$ des emplois en 2003), tandis que les emplois tertiaires de l'hôtellerie, du service ou la banque renforcent les petits centres urbains des vallées et des plaines. Mais si les flux touristiques sont en légère augmentation depuis 1991 (Tableau 6), le nombre d'hôtels diminue. Au total, les emplois liés au tourisme ne compensent pas ceux perdus par l'agriculture et l'industrie.

Tableau 4: Légère hausse des flux touristiques et concentration des installations dans les principales municipalités touristiques de montagne (Bohinj, Radovljica, Kranjka-gora, Bovec, Kobarid, Trzic)

\begin{tabular}{||c||c|c||c||c||c|c|}
\hline & \multicolumn{2}{|c|}{ Flux touristiques } & \multicolumn{2}{c|}{ Nombre de nuitées } & \multicolumn{2}{c|}{ Nombre d'hôtels } \\
\hline \hline $\mathbf{1 9 9 1}$ & 4121315 & Variation & 1241420 & Variation & 284 & Variation \\
\hline $\mathbf{1 9 9 5}$ & 3410091 & $-17 \%$ & 1137335 & $-8,4 \%$ & 233 & $-18 \%$ \\
\hline $\mathbf{2 0 0 1}$ & 4345960 & $+27,5 \%$ & 1351850 & $+18,9$ & 213 & $-9,5 \%$ \\
\hline
\end{tabular}

Parallèlement, les paysages se transforment. Les espaces agricoles sont peu à peu colonisés par les résidences secondaires. Grand nombre de bâtiments sont rachetés par des citadins non exploitants, et convertis en lieux de vacances. L'agriculture change de fonction, sa productivité décline au profit de l'entretien des paysages. Les opérations de valorisation patrimoniale se multiplient, les anciennes résidences des aristocrates de l'Empire austrohongrois sont réaménagées pour le tourisme. Les résidences estivales au bord du lac Bled, sont devenues aujourd'hui des hôtels de luxe. 
Pourtant, hors des sites touristiques, les paysages sont parsemés de petits centres industriels délaissés, tandis que les friches et la forêt gagnent du terrain. En dépit du temps radieux lors de mon dernier séjour sur place l'été 2003 et des hordes de touristes autrichiens venus chercher la fraîcheur en altitude, une impression lancinante de sinistre se dégage des lieux.

\section{Une redistribution spatiale des populations montagnardes}

Les mutations fonctionnelles de l'espace slovène interfèrent sur la distribution des populations en montagne. Le solde migratoire des régions alpine et préalpine est globalement positif ${ }^{5}$ (Carte 3) et bénéficie d'un net rajeunissement de la population, malgré le déficit d'emplois. Il est dû à un repeuplement soutenu par la croissance des petites villes de vallées, comme Kranj ou Skofja Loka, qui concentrent les nouveaux emplois tertiaires, mais surtout qui profitent de la répulsion de la capitale. L'étalement urbain très présent dans les plaines alpines, traduit une périurbanisation qui gagne actuellement la moyenne montagne, d'autant que les infrastructures routières s'améliorent.

En haute montagne, les nouveaux arrivants, souvent des professionnels du tourisme, des retraités, des résidents temporaires autrichiens pour la plupart, s'installent autour des principales stations touristiques, Bovec ou Bohinj par exemple. Le tourisme est désormais le moteur fondamental de l'essor démographique. Le parc national du Triglav, frontalier de l'Italie et de l'Autriche, pays à fort pouvoir d'achat, est la deuxième destination touristique après le littoral Adriatique. Les sports de plein air sont très développés en hiver comme en été, avec 23 stations de ski, 3 golfs, 5 centres équestres. Les aménagements autour des lacs de Bled et de Bohinj, à une heure de route de Ljubljana, tels que sentiers ou pannes pour les canoës, sont nombreux. Notons qu'entre 1999 et 2002, le fléchissement du solde migratoire dans ces régions laisse pressentir les limites du processus.

Aujourd'hui, en dépit d'un gain récent du peuplement en montagne, les contrastes démographiques s'accentuent. La population se concentre spécifiquement dans les petites villes et les stations touristiques tandis que les espaces restés à vocation rurale continuent à se vider.

\section{Conclusion}

La région alpine fait face depuis dix ans à des transformations radicales : bouleversements économiques, crise agricole et industrielle, tertiarisation, transformations paysagères, modification du marché et des secteurs de l'emploi. Les régions de montagne ne sortent pas gagnantes des transformations de la dernière décennie mais se réorganisent. Les mouvements de population sont nombreux et l'occupation de l'espace devient est de plus en plus contrastée. Que deviendront les villages ruraux qui continuent à se dépeupler ? La plaine serat-elle de plus en plus urbanisée ? Comment seront gérés les conflits émergents entre agriculture et habitat pour l'accès à la propriété foncière ? Quel est l'avenir de l'agriculture de montagne ?

\footnotetext{
5 Statistical Yearbook, 2000-2002, Statisticni urad Republike Slovenije (Statistical Office of the Republic of Slovenia), Ljubljana.
} 


\section{Bibliographie:}

Gabrovec M., Kladnik D., (1997), «Some New Aspects of Land Use in Slovenia», Acta Geographica Slovenica, ${ }^{\circ}$ 37, pp 8-63

Gabrovec M., Hrvatin M, (1998), Geographical Atlas of Slovenia, Ljubljana, 1998,p.83

Kladnik D., (1985), «Problematika zemljiske strukture v Sloveniji. Elaborat, 192 strani.», Institut za geografijo Univerze Edvarda Kardelja v Lubljana,

Klemenčič M., (1995), «La Slovénie subalpine, entre renaissance et déclin», Revue de géographie alpine, $\mathrm{n}^{\circ} 3$, tome 83 , pp. 39-50

Meze D., (1984), «Mountain farms in Slovenia», Geographica Iugoslavice, 5, pp. 47-55

Meze D., (1987), «Mountain farms in the Idrija and Cerklje regions», Geografski zbornik, 27, pp.5-67

Pechoux P.Y., Roux M., (1978), «Evolution récente de la moyenne montagne dans la péninsule balkanique. » Actes du 102eme Congrès national des sociétés savantes, Bibliothèque Nationale, Paris, pp 26-35

Pechoux P.Y., Roux M., (1983), «Evolution de la population de la Yougoslavie 1971$1981 »$, Méditerranée n4, pp27-34

Petek F., (2002), «Methodology of evaluation of changes in land use in Slovenia between 1896 and 1999», Acta Geographica Slovenica, n 42, pp.8-45

Vrišer I., (2002), «Agricultural production in the Republic of Slovenia (according to the census of the agricultural sector 2000)», Acta Geographica Slovenica n ${ }^{\circ} 42$, pp. 8-60

Statistical Yearbook, 1991, 1995, 2000, 2001, Statisticni urad Republike Slovenije, Ljubljana

Census of agriculture 1991 et 2000 Statisticni urad Republike Slovenije, Ljubljana. 